\author{
Frühe Folgeschäden
}

\title{
Kinder mit Typ-2-Diabetes besonders gefährdet
}

Eine Diabeteserkrankung in der Kindheit ist oft schon im jungen Erwachsenenalter mit Komplikationen und Begleiterkrankungen assoziiert. Das gilt für Typ-2- noch mehr als für Typ-1-Diabetiker, wie USamerikanische Diabetologen berichten. 1.746 Kinder mit Typ-1- und 272 mit Typ2-Diabetes wurden acht Jahre lang beobachtet. Typ-2-Diabetiker waren nicht nur viel häufiger adipös, sie litten auch häufi- ger schon an mikrovaskulären Diabeteskomplikationen und Komorbiditäten: Eine Nephropathie bestand bei 19,9\% gegenüber 5,8\% der Typ-1-Patienten, eine Retinopathie bei $9,1 \%$ vs. $5,6 \%$ und eine periphere Neuropathie bei $17,7 \%$ vs. $8,5 \%$. Ein Bluthochdruck wurde bei $21,6 \%$ vs. $10,1 \%$ festgestellt.

- JAMA 2017;317(8):825-835. doi:10.1001/jama.2017.0686; online 27. Februar 2017

\section{Inzidenzanstieg}

\section{Mehr subdurale Hämatome unter Antithrombotika}

Patienten mit gerinnungshemmender Therapie erleiden häufiger subdurale Blutungen, wie eine Fall-Kontroll-Studie aus Dänemark zeigt. „Fälle“ waren 10.010 Patienten, bei denen zwischen 2000 und 2015 ein Subduralhämatom diagnostiziert worden war. Als Kontrollen dienten 400.380 Personen aus der Allgemeinbevölkerung. Von den Patienten mit subduraler Blutung stan- den 47,3\% unter gerinnungshemmender Therapie. Für jede Art von Antihrombotikum wurde ein erhöhtes Risiko für Subduralhämatome ausgemacht: Unter ASS erhöhte sich das Risiko um 24\%, unter Clopidogrel um $87 \%$, unter einem DOAK um 73\%. Mit Vitamin-K-Antagonisten war das Risiko sogar mehr als verdreifacht (369\%). - JAMA 2017;317(8):836-8462

\section{Phytoöstrogene}

\section{Auf der sicheren Seite}

\section{Nasen mit abgekoch- tem Wasser duschen!}

In den vergangenen Jahren sind einige Berichte über tödlich verlaufene primäre Amöben-Meningoenzephalitiden nach Nasenspülungen erschienen. HNO-Spezialisten aus den USA prüften deshalb geeignete Vorsichtsmaßnahmen. Sie versetzten Wasser mit verschiedenen Keimen (u.a. Staph. aureus, Pseudomonas aeruginosa, Legionella pneumophila und Naegleria fowleri) und testeten drei Methoden zur Sterilisierung: Filtern durch einen Kohlenwasserstofffilter, Bestrahlung mit UVLicht und Abkochen. Der Filter konnte die Erreger nicht vollständig eliminieren. Bestrahlung, aber auch einfaches, fünfminütiges Kochen hingegen sterilisierte das Wasser.

- Laryngoscope 2017, online 22. Februar; doi: 10.1002/ lary. 26538

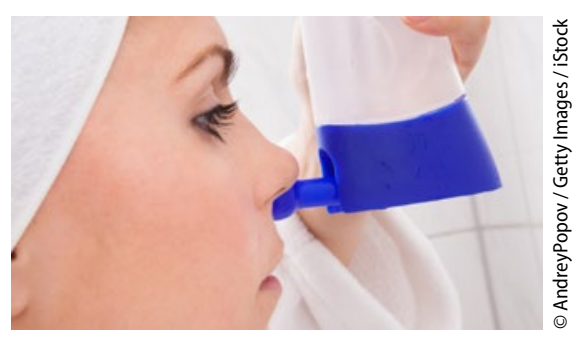

\section{Verlängert Soja das Überleben bei Brustkrebs?}

Isoflavone aus Soja haben möglicherweise bei manchen Brustkrebspatientinnen eine lebensverlängernde Wirkung. 6.235 Frauen, die im Breast Cancer Family Registry erfasst worden waren, hatten Angaben zu ihren Ernährungsgewohnheiten gemacht. Die mittlere Isoflavonaufnahme betrug 1,8 mg pro Tag. Im Laufe von rund neun Jahren starben 1.224 Patientinnen. Bei den Frauen im höchsten Isoflavonquartil (> 1,5 $\mathrm{mg} / \mathrm{d}$ ) war die Mortalität um $21 \%$ geringer als im untersten Quartil (<0,3 mg/d). Der Überlebensvorteil war allerdings auf Patientinnen mit hormonrezeptornegativen Tumoren bzw. ohne Hormontherapie beschränkt.

- Zhang F. F. et al. Cancer 2017, online 6. März

\section{Nächste Woche: CME- Sonderheft zum 500. Jubiläum}

In der nächsten Ausgabe haben wir wieder ein attraktives Paket von insgesamt sechs CME-Arbeiten für Sie geschnürt. Die Themen reichen von der ambulanten Versorgung Geflüchteter über die aktuellen Leitlinien bei akuten Atemwegsinfektionen bis zur "Schmerzenden Hüfte", Morbus Parkinson und Depressionen.

Übrigens: Diese Ausgabe enthält die 500. Folge der zertifizierten Fortbildung in der MMW. Seien Sie dabei! 